\title{
Pattern formation in a polymer thin film induced by an in-plane electric field
}

\author{
David Salac, Wei Lu, ${ }^{\text {a) }}$ Chia-Wei Wang, and Ann Marie Sastry \\ Department of Mechanical Engineering, University of Michigan, Ann Arbor, Michigan 48109
}

(Received 23 February 2004; accepted 21 June 2004)

\begin{abstract}
This letter reports experimental work involving use of an in-plane electric field to induce morphological patterns in a thin polymer film. The film was first spin coated onto a glass wafer. Then, it was heated to above its glass transition temperature to achieve mobility in the fluid. An in-plane electric field was applied using two parallel electrodes, spaced $10 \mathrm{~mm}$ apart, whereupon the initially flat polymer/air interface lost stability and formed islands. The self-assembled islands exhibited a narrow size distribution and demonstrated spatial ordering. We attribute the pattern formation to a combined mechanism of minimization of combined interface energy and electrostatic energy. () 2004 American Institute of Physics. [DOI: 10.1063/1.1781751]
\end{abstract}

Polymeric thin films enable manufacture of a wide variety of nanostructured devices and templates from which other nanofeatured materials can be created. Established techniques include block copolymer self-assembly, ${ }^{1}$ nanoimprint lithography, ${ }^{2}$ and soft lithography, ${ }^{2}$ among others.

One promising fabrication technique reported on recently is lithographically induced self-assembly (LISA) ${ }^{3-6}$ LISA is a process that utilizes the concept of electrohydrodynamic instabilities of polymers when exposed to an electric field. In instances of LISA, a flat thin polymer layer was first applied to a substrate using a technique such as spin coating. An upper mask is then set at a fixed distance (a few hundred nanometers) above the film, forming a small air gap. Between the substrate and the mask, an electric field perpendicular to the film surface is developed from either image charges $^{3,4}$ or external power supply., ${ }^{5,6}$ This causes pressure buildup at the polymer/air interface, and subsequent destabilization of the thin film. As a result, columns are formed toward the upper mask. It is also possible to direct this assembly using a topographically structured top mask, ${ }^{6}$ thus reproducing the mask pattern. Various orderings of columns have been constructed between the substrates and the masks, for example, the use of polymer/polymer/air trilayers have yielded structures comprised of cylinders having polymer cores surrounded by another polymer. ${ }^{6}$ Removal of the core, via selective etching, results in an ordered structure comprised of hollow cylinders.

Thus, in the LISA process, polymer structures are determined by the topographical patterns on the mask, making the technique well suited to pattern replication. However, the requirement of close placement of the upper mask relative to substrate complicates the production of large or curved surfaces. Specifically, the mask surface must be treated to prevent polymer adherence, so that the fine structures induced are not damaged during mask removal.

Here, we describe our recent experiment, in which we have used an electric field applied in the plane of the air/ polymer interface, instead of perpendicular to the interface. This setup greatly simplifies production, since no mask is

\footnotetext{
a) Author to whom correspondence should be addressed; electronic mail: weilu@umich.edu
}

required to produce fine features; instead, the morphological features self-assemble.

Figure 1 shows a schematic of the experimental setup, consisting of a pedestal, a pair of electrodes, a Pyrex riser, a substrate, and the polymer film. The pedestal was constructed from virgin electrical grade polytetrafluoroethylene. Besides providing support for the substrate and electrodes, it insulates the platform from other components. PTFE has high electrical resistivity, high dielectric strength, and can withstand high temperature. On the pedestal surface, two grooves were machined $10 \mathrm{~mm}$ apart to hold the electrodes tightly. The electrodes also had two grooves on the inner surface to secure the Pyrex riser, which raised the sample above the pedestal to allow rapid cooling. The electrodes were connected to a Glassman high-voltage power supply, capable of up to $125 \mathrm{kV}$. Thus, the average electric-field strength could reach $1.25 \times 10^{7} \mathrm{~V} / \mathrm{m}$.

Each sample consisted of a substrate and a spin-coated thin film. The substrates were made of Pyrex glass wafer, with dimensions of $10 \mathrm{~mm}$ by $30 \mathrm{~mm}$. They were thoroughly cleaned with toluene to remove dust and any organic compounds before spin coating. The setup in Fig. 1 was enclosed in an aluminum oven equipped with a front observation window. The outside of the aluminum enclosure was covered with a layer of thermal insulating material. A temperature controller and a thermocouple controlled the temperature inside. The operational temperature of the oven ranged between $300 \mathrm{~K}$ (room temperature) and $600 \mathrm{~K}$. The aluminum enclosure protected the environment from the high-voltage inside.

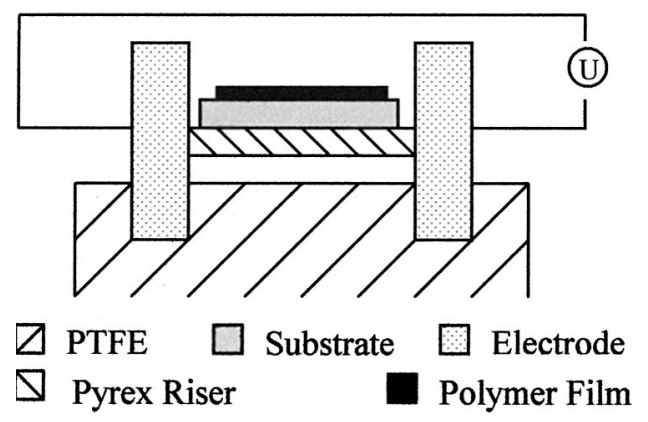

FIG. 1. A schematic of the experimental setup. 


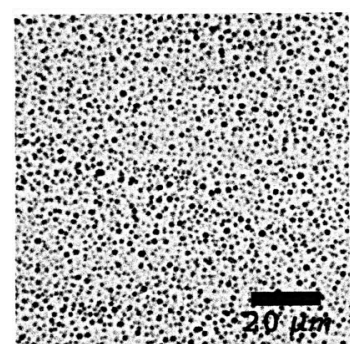

(a)

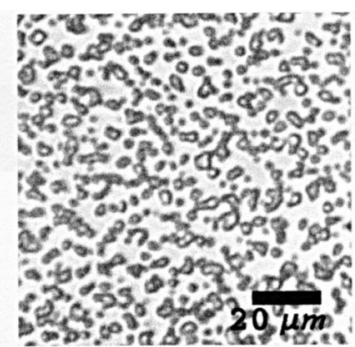

(b)
FIG. 2. Two optical images showing the overall morphology. (a) The polymer thin film is prepared from a $10 \%$ solution. (b) The polymer thin film is prepared from a $5 \%$ solution.

The polymer used in our experiment was polystyrene (PS). The properties of PS are well known, and those relevant to the experiment include: Molecular weight: $108 \mathrm{~kg} / \mathrm{mol}$; glass transition temperature: $100{ }^{\circ} \mathrm{C}$; surface tension: $30 \mathrm{mN} / \mathrm{m}$; and dielectric constant: 2.5 . PS can be easily spin coated onto a substrate. Each prepared sample was heated to $160{ }^{\circ} \mathrm{C}$, above the glass transition temperature of PS. An electrical voltage of $9.5 \mathrm{kV}$ was applied, resulting in an electric field with an average strength of $10^{6} \mathrm{~V} / \mathrm{m}$. The sample was left in these conditions for times ranging from 6 to $96 \mathrm{~h}$. After annealing, the sample was cooled to below the glass transition temperature and then the electric field was removed. During each experiment, a control sample was also utilized. A control sample experienced the same temperature and time condition, but was not exposed to any electric field. The samples were observed under both an optical microscope and an atomic force microscope (AFM) to investigate the overall patterns as well as the detailed features.

Two typical experimental results are shown in Figs. 2-4. In the first experiment, the substrate was spin coated with a $10 \%$ PS solution. The second experiment used a 5\% PS solution. They were both annealed for $96 \mathrm{~h}$. Figure 2 is an optical micrograph showing overall morphology. It was observed that patterns consisting of islands formed, in both cases. No structure formations were observed in any control

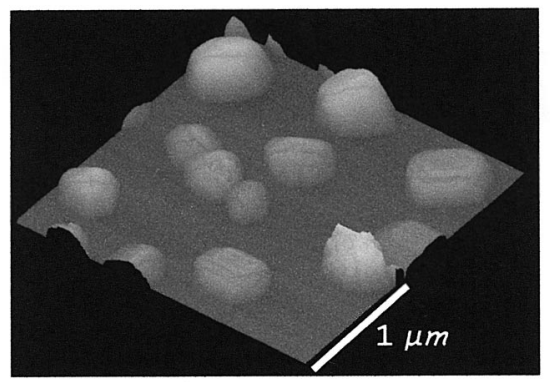

(a)

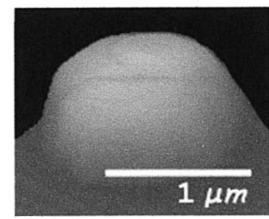

(b)

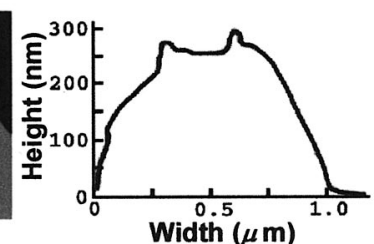

(c)
FIG. 3. The AFM images showing the morphology (a) $5 \mu \mathrm{m}$ by $5 \mu \mathrm{m}$ area. (b) A closer image of an island and (c) height profile. $10 \%$ solution.

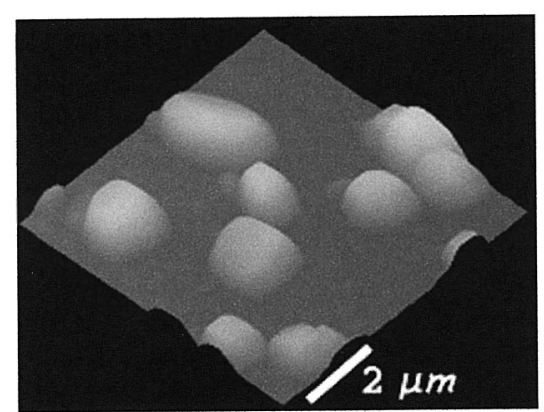

(a)

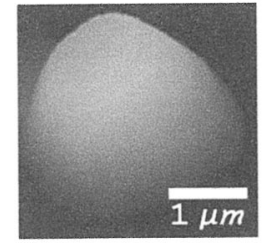

(b)

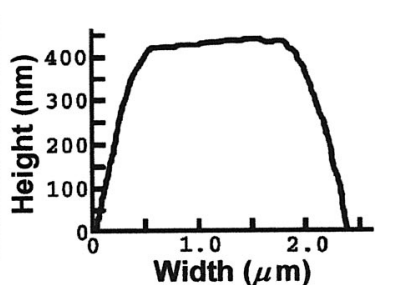

(c)
FIG. 4. The AFM images showing the morphology (a) $10 \mu \mathrm{m}$ by $10 \mu \mathrm{m}$ area. (b) A closer image of an island and (c) height profile. 5\% solution.

sample. Thus, we concluded that the electric field is the driving force behind this formation.

A difference in morphology can be observed in the two images in Fig. 2. Figure 2(a) (10\% solution) shows a closepacked hexagonal pattern, with a narrow size distribution of islands. Figure 2(b) (5\% solution) has a larger characteristic feature size. Also, some islands seem to have agglomerated into strips. Figure 3 (10\% solution) shows a physical characterization of the surface structures, obtained using atomic force microscopy in tapping mode. The diameters of most islands ranged from $1.2 \mu \mathrm{m}$ to $1.6 \mu \mathrm{m}$. Some smaller islands have diameters of about $300 \mathrm{~nm}$. The height of the islands averaged about $300 \mathrm{~nm}$. Figure 4 (5\% solution) shows an average diameter of features of approximately $2.5 \mu \mathrm{m}$ and an average height of roughly $400 \mathrm{~nm}$.

The cause of the observed differences in structure density, diameters, heights, and distributions is not yet known, though we postulate that the difference in feature size is due to the film thickness and nonlinearity in the late evolution process. A 5\% solution produces a thinner polymer film, and thus less material per unit area, than a film produced from a $10 \%$ solution. Imagine a small perturbation for two film thicknesses. The initial stage of evolution is quite similar in terms of linear stability analysis. However, the formation of islands in a thinner film depletes neighboring materials and starts nonlinear evolution earlier in the process. In the final stage, morphology significantly changes the local electricfield distribution. The islands in a thinner film do not have a chance to refine as much as they do in a thicker film. We plan to conduct experiments and nonlinear simulations as part of future research to better understand this phenomenon.

Theoretically, the origin of the film instability can be understood by considering the competition of forces acting at the polymer/air interface. ${ }^{7-9}$ The surface tension tends to minimize the polymer/air interface and stabilize a flat film. By contrast, the electric field induces an electrostatic pressure at the interface that destabilizes the film. This balance of forces can be written in terms of pressures, as 


$$
p=p_{0}-\gamma \frac{\partial^{2} h}{d x^{2}}+p_{\mathrm{el}},
$$

where $p_{0}$ is the atmospheric pressure, $h(x)$ is the film thickness, $\gamma$ is the surface tension, and $p_{\mathrm{el}}$ is the electrostatic pressure. The pressure induces a Poiseuille flow ${ }^{10}$

$$
j=\frac{h^{3}}{3 \mu}\left(-\frac{\partial p}{\partial x}\right),
$$

where $\mu$ is the temperature-dependent viscosity of the polymer melt. Mass conservation for the incompressible polymer melt requires

$$
\frac{\partial j}{\partial x}+\frac{\partial h}{\partial t}=0 .
$$

The pattern formation process at the polymer/air interface is described by Eqs. (1)-(3). A linear perturbation analysis gives the fastest growing wavelength by $\lambda_{m}$ $=2 \pi \sqrt{2 \gamma /\left(-\partial p_{\mathrm{el}} / \partial h\right)}$. Thus, an increase of electric field will reduce the feature size. With an in-plane electric field, the morphological change at one point will influence the entire domain. This global influence is desired as it allows structures at long distance sense each other, which may lead to patterns with better uniformity. Further work will explore this possibility.

${ }^{1}$ T. B. Liu, C. Burger, and B. Chu, Prog. Polym. Sci. 28, 5 (2003).

${ }^{2}$ Y. Xia, J. A. Rogers, K. E. Paul, and G. M. Whitesides, Chem. Rev. (Washington, D.C.) 99, 1823 (1999).

${ }^{3}$ S. Y. Chou and L. Zhuan, J. Vac. Sci. Technol. B 17, 3197 (1999).

${ }^{4}$ S. Y. Chou, L. Zhuang, and L. J. Guo, Appl. Phys. Lett. 75, 1004 (1999).

${ }^{5}$ E. Schäffer, T. Thurn-Albrecht, T. P. Russell, and U. Steiner, Nature (London) 403, 874 (2000).

${ }^{6}$ M. D. Morariu, N. E. Voicu, E. Schaffer, Z. Q. Lin, T. P. Russell, and U. Steiner, Nat. Mater. 2, 48 (2003).

${ }^{7}$ E. Schäffer, T. Thurn-Albrecht, T. P. Russell, and U. Steiner, Europhys. Lett. 53, 518 (2001).

${ }^{8}$ Z. Suo and J. Liang, Appl. Phys. Lett. 78, 3971 (2001).

${ }^{9}$ L. Wu and S. Y. Chou, Appl. Phys. Lett. 82, 3200 (2003).

${ }^{10}$ A. Vrij, Faraday Soc 42, 23 (1966). 